\title{
BMJ Open Global epidemiology of septic shock: a protocol for a systematic review and meta-analysis
}

\author{
Frank Leonel Tianyi, ${ }^{1}$ Joel Noutakdie Tochie (D) , ${ }^{2}$ Celestin Danwang (D) ,3,4 \\ Aime Mbonda, ${ }^{5}$ Mazou N Temgoua, ${ }^{6}$ Sylvester Yari Mapoh, ${ }^{7}$ Njinkeng J Nkemngu, ${ }^{8}$ \\ Esther Tallah, ${ }^{9}$ Jean Joel Bigna (D) ${ }^{10}$
}

To cite: Tianyi FL, Tochie JN, Danwang C, et al. Global epidemiology of septic shock: a protocol for a systematic review and meta-analysis. BMJ Open 2019;9:e032289. doi:10.1136/ bmjopen-2019-032289

- Prepublication history and additional material for this paper are available online. To view these files, please visit the journal online (http://dx.doi. org/10.1136/bmjopen-2019032289).

Received 13 June 2019 Revised 16 September 2019 Accepted 18 0ctober 2019
Check for updates

(C) Author(s) (or their employer(s)) 2019. Re-use permitted under CC BY-NC. No commercial re-use. See rights and permissions. Published by BMJ.

For numbered affiliations see end of article.

Correspondence to Dr Joel Noutakdie Tochie; joeltochie@gmail.com

\section{ABSTRACT}

Background Septic shock is a life-threatening infection frequently responsible for hospital admissions or may be acquired as nosocomial infection in hospitalized patients with resultant significant morbidity and mortality . There is a dearth of data on a résumé and meta-analysis on the global epidemiology of this potentially deadly condition. Therefore, we propose the first systematic review to synthesize existing data on the global incidence, prevalence and case fatality rate of septic shock worldwide.

Methods We will include cross-sectional, case-control and cohort studies reporting on the incidence, and case fatality rate of septic shock. Electronic databases including PubMed, Embase, WHO Global Health Library and Web of Science will be searched for relevant records published between 1 January 2000 and 31 August 2019. Independents reviewers will perform study selection and data extraction, as well as assessment of methodological quality of included studies. Appropriate meta-analysis will then be used to pool studies judged to be clinically homogenous. Egger's test and funnel plots will be used to detect publication bias. Findings will be reported and compared by human development level of countries. Ethics and dissemination Being a review, ethical approval is not required as it was obtained in the primary study which will make up the review. This review is expected to provide relevant data to help in evaluating the burden of septic shock in the general population. The overall findings of this research will be published in a peerreviewed journal.

PROSPERO registration number CRD42019129783.

\section{INTRODUCTION}

According to Sepsis-3, the most recent international consensus on sepsis and septic shock (SS), SS is defined as a sepsis (denoted by a Sequential Organ Failure Assessment $\geq 2$ points) plus serum lactate $\geq 2 \mathrm{mmol} / \mathrm{L}$ and persistent hypotension despite adequate volume resuscitation, necessitating vasopressors to maintain a mean arterial blood pressure $\geq 65 \mathrm{~mm} \mathrm{Hg}^{1}{ }^{1}$ Globally, $\mathrm{SS}$ is one of the most common cause of admission in both the adult and paediatric populations ${ }^{23}$

\section{Strengths and limitations of this study}

- The restriction of septic shock (SS) definitions to the Sepsis-3 consensus and the International Pediatric Sepsis Consensus for adults and children, respectively, will limit major heterogeneity.

- Robust methods and statistical analyses such as meta-analysis will be used to determine the global burden of SS.

- The present review will include primary studies without language restrictions, and thus will allow us to enrol the maximum of studies published and unpublished on the subject.

- A limited number of studies on the topic in lowincome and middle-income countries could lead to an underestimation of the true global burden of SS.Any significant heterogeneity in the included studies may preclude the pooling of data to perform a meta-analysis

and it is associated with a significantly high mortality rate. ${ }^{3-7} \mathrm{SS}$ is globally recognised by WHO as a health priority. ${ }^{8}$ In patients with SS, the blood perfusion of noble organs is compromised, leading to encephalopathies, acute respiratory distress syndrome, acute hepatic failure, ischaemic heart disease, sepsis-induced coagulopathy, multiorgan dysfunction and ultimately death if not timely and appropriately treated. ${ }^{9}{ }^{10}$ Survivors often have a reduced quality of life due to long-term cognitive, psychological, physical sequelae ${ }^{1112}$ and equally have a higher risk for 1-year mortality following hospital discharge for SS. ${ }^{13}$ Antibiotics play a key role in the pharmacological management of SS; however, the timing of initiation of antibiotic therapy still remains an important debate in clinical practice. Furthermore, the management of SS currently challenging in identifying the correct hemodynamic control, optimisation of fluid resuscitation, choosing the appropriate vasopressors, triage of 
patients who need ionotropic drugs, beta-blockers and steroids. ${ }^{14}$

Some multinational studies on the global prevalence of sepsis but not SS found a prevalence rate varying between $29.5 \%$ and $51 \% .{ }^{6}{ }^{15} \mathrm{~A}$ more systematic review found that the frequency and mortality rate of SS stood between $8.3 \%$ to $10.4 \%$ and $37.3 \%$, respectively, in both Europe and North America combined. ${ }^{16}$ However, there is a dearth of contemporary data on the global epidemiological of SS, making it impossible to appraise the burden of SS on a global perspective. Despite this gap in knowledge on the topic, currently, no research has highlighted the global epidemiology of SS. Accordingly, we propose this systematic review and meta-analysis protocol to critically synthesize contemporary evidence on the occurrence of SS in order to provide a clear understanding of its up-todate burden in the world. The research goal being to provide useful data that may help guide resource allocation by informing health authorities.

\section{REVIEW QUESTIONS}

What is the burden of SS in the global population?

\section{OBJECTIVE}

The aim of this systematic review and meta-analysis is to determine the global incidence, and case fatality rate of SS on a global basis.

\section{METHODS}

This review is reported in accordance with the Preferred Reporting Items for Systematic Review and Meta-Analysis Protocols 2015 Guidelines ${ }^{17}$ and applicable to observational studies. This is illustrated in more detail in online additional file 1 .

\section{Eligibility criteria}

Types of studies

We will include cross-sectional, case-control and cohort studies. Commentaries, editorials, letters and reviews will not be considered. Studies with inaccessible full texts either online or from the corresponding author will be excluded.

\section{Types of patients}

We will consider studies including hospitalised adults and children.

\section{Types of outcomes}

The diagnosis of SS in adults will be based on the Sepsis-3 Consensus, ${ }^{1}$ whereas SS in children will be defined in conformity with the International Pediatric Sepsis Consensus $^{18}$ (online additional 2). The incidence of SS will be defined number of individuals who developed SS between 1 January 2000 and 31 August 2019. We will exclude studies in which relevant data on patients with SS are impossible to extract even after contacting the corresponding author.

Search strategy for identifying relevant studies

The search strategy will be as follows:

Bibliographic database searches: PubMed, Embase, WHO Global Health Library and Web of Science will be searched for relevant records published in English or French between 1 January 2000 and 31 August 2019. The designed search strategy for PubMed using both text words and medical subject heading terms related to sepsis, septicaemia, bacteraemia, infection and shock is provided in online additional file 3 . This search strategy will be adapted to fit with other databases.

\section{Searching for other sources}

We will scan the references of all relevant articles for additional data sources missed during our search strategy, and their full texts will be sorted. Citations of important reviews will also be scanned. Lastly, the search strategy will extend to include grey literature from conference proceedings, book chapters, theses, government and non-governmental organisations reports.

\section{Selection of studies for inclusion in the review}

Two reviewers (FLT and CD) will independently evaluate the records obtained from the searching process, with the aid of an evaluation form to ensure reliably application of the selection criteria. These reviewers will screen the titles and abstracts of records obtained. Next, the full texts of potentially eligible articles will be retrieved by at least one author. The two reviewers will independently review the full text of each potentially eligible article, compare their findings and resolve any discordance by the arbitration of a third author (MNT). For duplicates articles, only the study reporting the largest sample size will be considered.

\section{Data extraction and management}

A data extraction form will be used by three pairs of independent reviewers (JNT and AM), (FLT and CD) and (SYM and MNT) to collect information on the last name of the first author, year of publication, region, country name, human development index ranking of country economic level, study area (rural vs urban), study setting, number of sites, study design, mean or median age, sampling method, sample size, timing of data collection, male proportion, specific characteristics of the study population (patients with HIV, cancer, diabetes mellitus, organ transplants or any other specific condition), incidence rate, site of infection, Sequential Organ Failure Assessment (SOFA), serum late level in $\mathrm{mmol} / \mathrm{L}$, bacteria involved in the infection and case fatality rate of SS. For multicentre studies conducted in different countries, the incidence, prevalence and case fatality rate will be reported separately for the individual countries.

\section{Data synthesis and analysis}

After data collection, a meta-analysis will be conducted when there will be clinical homogeneity based on the 
profile of the population. Unadjusted SE, case fatality rate, prevalence and incidence for the study-specific estimates will be resumed based on the crude information of the numerators, and denominators provided by each study. Subgroup analyses will be performed by separate pooling of studies conducted among children and adolescents. To maintain the effect of studies with extremely small or large estimates on the overall estimate to a minimum, the variance of the study-specific incidence/case fatality rate will be stabilised with the Freeman-Tukey double arcsine transformation ${ }^{19}$, before pooling the data using a random effects meta-analysis model. Heterogeneity will be assessed using the $\chi^{2}$ test on Cochrane's $Q$ statistic and quantified by calculating $\mathrm{I}^{2} .{ }^{20}$ Values of $25 \%, 50 \%$ and $75 \%$ for $\mathrm{I}^{2}$ will, respectively, represent low, medium and high heterogeneity. We will assess the presence of publication bias using funnel plots inspection and Egger's test. ${ }^{21}$ Where substantial heterogeneity will be detected, metaregression and subgroup analyses will be performed to investigate the possible sources of heterogeneity using the aforementioned variables and the study methodological quality. In case of substantial clinical heterogeneity, a narrative summary of our findings will be done. The inter-rater agreement for study inclusion between investigators will be assessed using Cohen's k coefficient. ${ }^{22}$ Data analyses will be done using the 'meta' package of the statistical software R (V.3.5.1, The R Foundation for Statistical Computing, Vienna, Austria).

\section{Presentation and reporting of results}

The study selection process will be summarized in a flow diagram. Quantitative data will be presented in evidence tables of individual studies as well as in summary tables and forest plots where appropriate. The quality scores and risk of bias for each eligible study will be reported accordingly. This may be tabulated and accompanied by narrative summaries.

\section{Patient and public involvement}

In this study, data will be collected directly from published articles available in main databases and unpublished studies. Patient and public are not involved in the development of this protocol.

\section{Potential amendments}

Amendments made include broadening the scope of the study to a global epidemiology of SS; not synthesising the risk factors and prevalence of SS anymore. The definitions of SS will be based on the Sepsis-3 Consensus and the International Pediatric Sepsis Consensus for adults and children, respectively. These amendments will be updated in the review submitted to the International Prospective Resgister for Systematic Reviews (PROSPERO) under the number CRD42019129783 for better transparency.

\section{ETHICS AND DISSEMINATIONS}

The current review will use published studies. Therefore, there is no requirement for ethical approval. The review is expected to provide the current global burden of SS in the global population of inpatients in order to inform health authorities and decision makers to elaborate effective preventive strategies to reduce the burden of SS in this high-risk patient population. The resulting manuscript will be published in a peer-reviewed journal and presented at scientific conferences.

\section{Review status}

Preliminary searches.

\section{Author affiliations}

${ }^{1}$ Department of General Medicne, Mayo Darle Sub-Divisional Hospital, Banyo, Adamawa, Cameroon

${ }^{2}$ Department of Anaesthesiology and Critical Care Medicine, Faculty of Medicine and Biomedical Sciences, University of Yaounde 1, Yaounde, Cameroon

${ }^{3}$ School of Public Health, Universite Libre de Bruxelles, Bruxelles, Belgium ${ }^{4}$ Department of Surgery and sub-Specialties, Faculty of Medicine and Biomedical Sciences, University of Yaounde 1, Yaounde, Cameroon

${ }^{5}$ Department of Surgery, National Social Insurance Fond Hospital, Yaounde, Cameroon

${ }^{6}$ Department of Internal Medicine and sub-Specialties, Faculty of Medicine and Biomedical Sciences, University of Yaounde 1, Yaounde, Cameroon

${ }^{7}$ Department of Family Medicine, University of Louisiana, New Orleans, Louisiana, USA

${ }^{8}$ Department of Anesthesia, University of Toronto, Toronto, Ontario, Canada

${ }^{9}$ Cameroon Coalition Against Malaria, Yaounde, Cameroon

${ }^{10}$ Department of Epidemiology and Public Health, Centre Pasteur of Cameroon, Yaoundé, Cameroon

\section{Twitter Frank Leonel Tianyi @tianyifrank1}

Contributors FLT, JNT and NJN had the idea, designed and conceived the protocol. JNT wrote the first draft of the manuscript. JNT, FLT, CB and JJB are the guarantors of the review. All authors critically revised the methodology and intellectual content and approved the final version of this manuscript.

Funding The authors have not declared a specific grant for this research from any funding agency in the public, commercial or not-for-profit sectors.

Competing interests None declared.

Patient consent for publication Not required.

Provenance and peer review Not commissioned; externally peer reviewed.

Open access This is an open access article distributed in accordance with the Creative Commons Attribution Non Commercial (CC BY-NC 4.0) license, which permits others to distribute, remix, adapt, build upon this work non-commercially, and license their derivative works on different terms, provided the original work is properly cited, appropriate credit is given, any changes made indicated, and the use is non-commercial. See: http://creativecommons.org/licenses/by-nc/4.0/.

\section{ORCID iDs}

Joel Noutakdie Tochie http://orcid.org/0000-0002-8338-2467

Celestin Danwang http://orcid.org/0000-0002-7976-4331

Jean Joel Bigna http://orcid.org/0000-0001-8018-6279

\section{REFERENCES}

1 Singer M, Deutschman CS, Seymour CW, et al. The third International consensus definitions for sepsis and septic shock (Sepsis-3). JAMA 2016;315:801-10.

2 Thompson K, Venkatesh B, Finfer S. Sepsis and septic shock: current approaches to management. Intern Med J 2019;49:160-70.

3 Fleischmann C, Scherag A, Adhikari NKJ, et al. Assessment of global incidence and mortality of hospital-treated sepsis. current estimates and limitations. Am J Respir Crit Care Med 2016;193:259-72.

4 Liu V, Escobar GJ, Greene JD, et al. Hospital deaths in patients with sepsis from 2 independent cohorts. JAMA 2014;312:90-2.

5 Shankar-Hari M, Phillips GS, Levy ML, et al. Developing a new definition and assessing new clinical criteria for septic shock: for the 
third International consensus definitions for sepsis and septic shock (Sepsis-3). JAMA 2016;315:775-87.

6 Sakr Y, Jaschinski U, Wittebole X, et al. Sepsis in intensive care unit patients: worldwide data from the intensive care over nations audit. Open Forum Infect Dis 2018;5.

7 Annane D, Aegerter P, Jars-Guincestre MC, et al. Current epidemiology of septic shock: the CUB-Réa network. Am J Respir Crit Care Med 2003;168:165-72.

8 Reinhart K, Daniels R, Kissoon N, et al. Recognizing sepsis as a global health priority - a who resolution. $N$ Engl $J$ Med 2017;377:414-7.

9 Balcan B, Olgun S, Torlak F, et al. Determination of factors affecting mortality of patients with sepsis in a tertiary intensive care unit. Turk Thorac J 2015;16:128-32.

10 Cohen J. The immunopathogenesis of sepsis. Nature 2002;420:885-91.

11 Yende S, Austin S, Rhodes A, et al. Long-term quality of life among survivors of severe sepsis: analyses of two international trials. Crit Care Med 2016;44:1461-7.

12 Iwashyna TJ, Ely EW, Smith DM, et al. Long-term cognitive impairment and functional disability among survivors of severe sepsis. JAMA 2010;304:1787-94.

13 Prescott HC, Osterholzer JJ, Langa KM, et al. Late mortality after sepsis: propensity matched cohort study. BMJ 2016;353.
14 De Backer D, Cecconi M, Lipman J, et al. Challenges in the management of septic shock: a narrative review. Intensive Care Med 2019;45:420-33.

15 Vincent J-L, Rello J, Marshall J, et al. International study of the prevalence and outcomes of infection in intensive care units. JAMA 2009;302:2323-9.

16 Vincent J-L, Jones G, David S, et al. Frequency and mortality of septic shock in Europe and North America: a systematic review and meta-analysis. Critical Care 2019;23.

17 Moher D, Shamseer L, Clarke M, et al. Preferred reporting items for systematic review and meta-analysis protocols (PRISMA-P) 2015 statement. Syst Rev 2015;4:1.

18 Goldstein B, Giroir B, Randolph A, et al. International pediatric sepsis consensus conference: definitions for sepsis and organ dysfunction in pediatrics. Pediatr Crit Care Med 2005;6:2-8.

19 Miller JJ. The inverse of the Freeman - Tukey double arcsine transformation. The American Statistician 1978;32:138.

20 Huedo-Medina TB, Sánchez-Meca J, Marín-Martínez F, et al. Assessing heterogeneity in meta-analysis: $\mathrm{Q}$ statistic or $\mathrm{I}^{2}$ index? Psychol Methods 2006;11:193-206.

21 Egger M, Davey Smith G, Schneider M, et al. Bias in meta-analysis detected by a simple, graphical test. BMJ 1997;315:629-34.

22 McHugh ML. Interrater reliability: the kappa statistic. Biochem Med 2012;22:276-82. 